\title{
CONSTRUCTING SEQUENCES OF DIVIDED POWERS. II
}

\author{
ASTRID J. BERKSON AND KENNETH NEWMAN ${ }^{1}$
}

\begin{abstract}
Using principally the exponential and logarithmic Taylor series a number of simple formulas are derived for extending sequences of divided powers in cocommutative irreducible Hopf algebras over fields of characteristic 0 . Both the commutative and noncommutative cases are considered.
\end{abstract}

Let $H$ be a cocommutative irreducible Hopf algebra over the rationals. A sequence of elements in $H, x_{0}=1, x_{1}, x_{2}, \ldots, x_{n}$ or $x_{0}=1, x_{1}, x_{2}, \ldots$ is called a sequence of divided powers if, for all $i$,

$$
\Delta x_{i}=\sum_{j=0}^{i} x_{j} \otimes x_{i-j}
$$

In [1] there are a number of theorems on the constructibility of sequences of divided powers in the case when $H$ is commutative. Here, we improve on the central result of that paper [Theorem 2, p. 33] and greatly simplify its proof. We also give generalizations to the noncommutative case.

The underlying computational technique, roughly stated, is that the exponential Taylor series of a primitive is a sequence of divided powers while the logarithmic Taylor series of a sequence of divided powers is a primitive. This allows us to give a simple and precise description of the form of an extension of a sequence of divided powers in both the commutative and noncommutative cases.

Throughout this paper, it will be assumed without reiteration that all Hopf algebras are cocommutative and irreducible and are over $\mathbf{Q}$, the rationals.

A formal power series in $T, 1+\sum_{i=1}^{\infty} x_{i} T^{i}$ will be called a sequence of divided powers (SDP) if $1, x_{1}, x_{2}, \ldots$ is an SDP. Note that if $1+\sum_{i=1}^{\infty} x_{i} T^{i}$ is an SDP then $1+\sum_{i=1}^{\infty} x_{i} T^{i n}$ is an SDP for all $n \geqslant 1$.

Lemma 1 [2, Sublemma, p. 28]. If $x_{0}=1, x_{1}, x_{2}, \ldots$ and $y_{0}=1, y_{1}$, $y_{2}, \ldots$, are both SDP's in the same Hopf algebra then $\left\{\sum_{j=0}^{i} x_{j} y_{i-j}\right\}_{i}$ is also an SDP. Equivalently, if $\left(1+\sum_{i=1}^{\infty} x_{i} T^{i}\right)$ and $\left(1+\sum_{i=1}^{\infty} y_{i} T^{i}\right)$ are SDP's, then $\left(1+\sum_{i=1}^{\infty} x_{i} T^{i}\right)\left(1+\sum_{i=1}^{\infty} y_{i} T^{i}\right)$ is an SDP.

Proposition 2. Let $x_{1}, x_{2}, \ldots$ be elements in a commutative Hopf algebra.

Recieved by the editors September 26, 1977.

AMS (MOS) subject classifications (1970). Primary 16A24.

Key words and phrases. Cocommutative irreducible Hopf algebras, sequence of divided powers.

'This research was partially supported by National Science Foundation Grant NSF 76-066 38A01.

(1) American Mathematical Society 1978 
Then the formal power series in $T, \exp \left(\sum_{i=1}^{\infty} x_{i} T^{i}\right)$, is an SDP if and only if each $x_{i}$ is primitive. (Note that 0 is a primitive.)

Proof. $\Leftarrow$ It is well known and easy to compute that $\exp \left(x T^{n}\right)=$ $\sum_{i=0}^{\infty}\left(x^{i} / i !\right) T^{i n}$ is an SDP if $x$ is a primitive. Consequently,

$$
\exp \left(\sum_{i=0}^{\infty} x_{i} T^{i}\right)=\prod_{i=1}^{\infty} \exp \left(x_{i} T^{i}\right)
$$

will be an SDP using the lemma and induction.

$\Rightarrow$ Assume inductively that $x_{i}$ is a primitive for all $i<n$. Let $y_{j}$ be the coefficient of $T^{j}$ in $\exp \left(\sum_{i=1}^{n-1} x_{i} T^{i}\right)$. We have just shown that $1, y_{1}, y_{2}, \ldots$ is an SDP. Note that in $\exp \left(\sum_{i=1}^{\infty} x_{i} T^{i}\right)$ the coefficient of $T^{j}$ is $y_{j}$ for $j<n$ and $y_{j}+x_{j}$ for $j=n$. Thus $y_{n}$ and $y_{n}+x_{n}$ both extend the same SDP which implies that their difference is a primitive.

Corollary 3. $1+\sum_{i=1}^{\infty} x_{i} T^{i}$ is an SDP if and only if the coefficient of $T^{j}$ in $\log \left(1+\sum_{i=1}^{\infty} x_{i} T^{i}\right)$ is a primitive for all $j$.

Proof. Since log and exp are inverse functions, this follows immediately.

TheOREM 4. Give $H_{n}=\mathbf{Q}\left[X_{1}, X_{2}, \ldots, X_{n}\right]$ a graded Hopf algebra structure by defining $\Delta X_{i}$ so that $1, X_{1}, X_{2}, \ldots, X_{n}$ is an SDP and by letting $\operatorname{deg} X_{i}=i$. Then

$$
P_{n+1}=\sum \frac{(-1) \sum m_{i}}{\sum m_{i}}\left(\begin{array}{c}
\sum m_{i} \\
m_{1}, m_{2}, \ldots, m_{n}
\end{array}\right) X_{1}^{m_{1}} X_{2}^{m_{2}} \cdots X_{n}^{m_{n}}
$$

(the outer sum is taken over all $m_{1}, m_{2}, \ldots, m_{n}$ such that $m_{1}+2 m_{2}$ $\left.+\cdots+n m_{n}=n+1\right)$ is the unique homogeneous extension to $1, X_{1}$, $X_{2}, \ldots, X_{n}$.

Proof. Consider $H=\mathrm{Q}\left[X_{1}, X_{2}, \ldots\right]$ where $1, X_{1}, X_{2}, \ldots$ is an SDP. By Corollary 3 , for all $j$, the coefficient of $T^{j}$ in

$$
\log \left(1+\sum_{i=1}^{\infty} X_{i} T^{i}\right)=\sum_{i=1}^{\infty} X_{i} T^{i}-\frac{\left(\sum_{i=1}^{\infty} X_{i} T^{i}\right)^{2}}{2}+\frac{\left(\sum_{i=1}^{\infty} X_{i} T^{i}\right)^{3}}{3}-\ldots
$$

is a primitive. In particular the coefficient of $T^{n+1}$

$$
\sum \frac{(-1)^{1+\Sigma m_{i}}}{\sum m_{i}}\left(\begin{array}{c}
\sum m_{i} \\
m_{1}, m_{2}, \ldots, m_{n+1}
\end{array}\right) X_{1}^{m_{1}} X_{2}^{m_{2}} \cdots X_{n+1}^{m_{n+1}}
$$

(the outer sum is taken over all $m_{1}, m_{2}, \ldots, m_{n+1}$ such that $m_{1}+2 m_{2}$ $\left.+\cdots+(n+1) m_{n+1}=n+1\right)$ is a primitive. Therefore $P_{n+1}$, the difference between $X_{n+1}$ and this polynomial, will be an extension of $1, X_{1}$, $X_{2}, \ldots, X_{n}$.

Uniqueness follows from [3, Lemma 4] or [1, Theorem 2, p. 33].

We turn now to the noncommutative case and show that Proposition 2 and 
Corollary 3 still hold. We will then be able to prove a theorem analogous to Theorem 4.

Proposition 5. Assume $1, x_{1}, x_{2}, \ldots$ is an SDP in a Hopf algebra $H$. Then for all $j \geqslant 1$ the coefficient of $T^{j}$ in $\log \left(1+\sum_{i=1}^{\infty} x_{i} T^{i}\right)$ is a primitive. (We assume of course that $T$ commutes with the $x_{i}$.)

Proof. Let $y_{j}$ be the coefficient of $T^{j}$ in $\log \left(1+\sum_{i=1}^{\infty} x_{i} T^{i}\right)$. Then

$$
y_{j}=\sum \frac{(-1)^{n+1}}{n} x_{i_{1}} x_{i_{2}} \cdots x_{i_{n}}
$$

summing over all terms such that $\sum i_{k}=j\left(1 \leqslant i_{k} \leqslant j\right)$. We wish to show that any term in $\Delta y_{j}$ of the form $x_{i_{1}} \cdots x_{i_{t}} \otimes x_{i_{t+1}} \cdots x_{i_{m}}$ with $t \geqslant 1$ and $m>t$ has coefficient 0 . We know (Corollary 3) that in the commutative case $x_{i_{1}} \cdots x_{i_{i}} \otimes x_{i_{i+1}} \cdots x_{i_{m}}$ has coefficient 0 . This means that the sum of the coefficients of all distinct terms of the form

$$
x_{i\langle(1)} \cdots x_{i_{\psi}(t)} \otimes x_{i_{\phi(t+1)}} \cdots x_{i_{\phi}(m)}
$$

where $\psi$ is a permutation of $\{1, \ldots, t\}$ and $\phi$ a permutation of $\{t+$ $1, \ldots, m\}$ must be zero. We will show that when distinct $x_{i}$ do not satisfy any nontrivial polynomial identity that these coefficients are all the same and thus all must be zero. (This will clearly imply the general case.) Actually we will show that the coefficient of $x_{i_{1}} \cdots x_{i_{t}} \otimes x_{i_{t+1}} \cdots x_{i_{m}}$ depends only on $t$ and $m$.

Consider all ways in which $x_{i_{1}} \cdots x_{i_{1}} \otimes x_{i_{i+1}} \cdots x_{i_{m}}$ can occur in $\Delta y_{j}$. If

$$
\left(x_{j_{1}} \otimes x_{k_{1}}\right)\left(x_{j_{2}} \otimes x_{k_{2}}\right) \cdots\left(x_{j_{n}} \otimes x_{k_{n}}\right)=x_{i_{1}} \cdots x_{i_{1}} \otimes x_{i_{1+1}} \cdots x_{i_{m}}
$$

then of course

$$
x_{j_{1}} \cdots x_{j_{n}}=x_{i_{1}} \cdots x_{i_{r}} \text { and } x_{k_{1}} \cdots x_{k_{n}}=x_{i_{t+1}} \cdots x_{i_{m}},
$$

i.e. each left-hand product must match each right-hand product in the same order, except that on the left-hand side there may be some l's i.e. we allow $j_{l}$ or $k_{l}$ to be zero, but we will assume that if $x_{j_{l}}=1$ then $x_{k_{l}} \neq 1$. Now $\left(x_{j_{1}} \otimes x_{k_{1}}\right) \cdots\left(x_{j_{n}} \otimes x_{k_{n}}\right)$ occurs only in $\Delta x_{j_{1}+k_{1}} \cdots x_{j_{n}+k_{n}}$ (and has coefficient 1) and $x_{j_{1}+k_{1}} \cdots x_{j_{n}+k_{n}}$ is a term in $y_{j}$ with coefficient $(-1)^{n+1} 1 / n$, i.e. $\left(x_{j_{1}} \otimes x_{k_{1}}\right) \cdots\left(x_{j_{n}} \otimes x_{k_{n}}\right)$ has coefficient $(-1)^{n+1} 1 / n$ in $\Delta y_{j}$. (In the above discussion when we say that $\left(x_{j_{1}} \otimes x_{k_{1}}\right) \cdots\left(x_{j_{n}} \otimes x_{k_{n}}\right)$ occurs only in $\Delta x_{j_{1}+k_{1}} \cdots x_{j_{n}+k_{n}}$ we mean before any multiplying is done. Thus, for example, $\left(1 \otimes x_{1}\right)\left(x_{2} \otimes 1\right)$ and $\left(x_{2} \otimes 1\right)\left(1 \otimes x_{1}\right)$ are, for the moment, to be thought of as distinct products. The former is a term in $\Delta x_{1} x_{2}$ and the latter a term in $\Delta x_{2} x_{1}$.) Now the coefficient of $x_{i_{1}} \cdots x_{i_{1}} \otimes$ $x_{i_{i+1}} \cdots x_{i_{m}}$ will be the sum of all coefficients of the products $\left(x_{j_{1}} \otimes\right.$ $\left.x_{k_{1}}\right) \cdots\left(x_{j_{n}} \otimes x_{k_{n}}\right)$. But clearly the number and form of these products is independent of which particular $x_{i}$ occur in $x_{i_{1}} \cdots x_{i_{i}} \otimes x_{i_{i+1}} \cdots x_{i_{m}}$ and the coefficient of each product depends only on $n$.

EXAMPLE. $x_{2} x_{1} \otimes x_{1}$ will occur in $\Delta y_{4}$ as 
$(1)\left(x_{2} \otimes 1\right)\left(x_{1} \otimes 1\right)\left(1 \otimes x_{1}\right)$,

(2) $\left(x_{2} \otimes 1\right)\left(1 \otimes x_{1}\right)\left(x_{1} \otimes 1\right)$,

(3) $\left(1 \otimes x_{1}\right)\left(x_{2} \otimes 1\right)\left(x_{1} \otimes 1\right)$,

(4) $\left(x_{2} \otimes 1\right)\left(x_{1} \otimes x_{1}\right)$,

$(5)\left(x_{2} \otimes x_{1}\right)\left(x_{1} \otimes 1\right)$.

The first three products, since they have three factors, will have coefficient $\frac{1}{3}$ while the latter two will have coefficient $-\frac{1}{2}$. Thus the coefficient of $x_{2} x_{1} \otimes x_{1}$ is $3\left(\frac{1}{3}\right)-2\left(\frac{1}{2}\right)$. Note that the fact that the first and second products both occur in $\Delta x_{2} x_{1} x_{1}$ is irrelevant. The point is that changing $x_{2}$ and/or either $x_{1}$ to any other $x_{i}$ would not affect the computation.

Proposition 6. If $x_{1}, x_{2}, \ldots$ are primitives in a Hopf algebra then $\exp \left(\sum_{i=1}^{\infty} x_{i} T^{i}\right)$ is an $S D P$.

Proof. We will find an SDP whose log equals $\sum_{i=1}^{\infty} x_{i} T^{i}$. Since log and exp are inverse functions this will suffice. Assume inductively that we have an SDP, $1+\sum_{i=1}^{\infty} z_{i} T^{i}$, such that the coefficient of $T^{j}$ in $\log \left(1+\sum_{i=1}^{\infty} z_{i} T^{i}\right)$ is $x_{j}$ for $1 \leqslant j<n$. (The induction can be started with $\exp \left(x_{1} T\right)$.) Let the coefficient of $T^{n}$ in $\log \left(1+\sum_{i=1}^{\infty} z_{i} T^{i}\right)$ be $w$. By Proposition $5 w$ is a primitive. Then $\left(1+\sum_{i=1}^{\infty} z_{i} T^{i}\right) \exp \left(\left(x_{n}-w\right) T^{n}\right)$ will be an SDP (Lemma 1) and will continue the induction.

THEOREM 7. Let $x_{1}, x_{2}, \ldots$ be elements of a Hopf algebra. Then

(a) $1+\sum_{i=1}^{\infty} x_{i} T^{i}$ is an SDP if and only if the coefficient of $T^{j}$ in $\log (1+$ $\left.\sum_{i=1}^{\infty} x_{i} T^{i}\right)$ is a primitive for all $j$.

(b) $\exp \left(\sum_{i=1}^{\infty} x_{i} T^{i}\right)$ is an SDP if and only if $x_{i}$ is a primitive for all $i$.

Proof. Follows from Propositions 5 and 6.

Theorem 8. Give $H_{n}=\mathbf{Q}\left\langle X_{1}, X_{2}, \ldots, X_{n}\right\rangle$ (the free algebra on $X_{1}$, $\left.X_{2}, \ldots, X_{n}\right)$ a Hopf algebra structure by defining $\Delta X_{i}$ so that $1, X_{1}, X_{2}, \ldots, X_{n}$ is an SDP. Then $\Sigma\left((-1)^{m} / m\right) X_{i_{1}} X_{i_{2}} \cdots X_{i_{m}}$ extends $1, X_{1}, X_{2}, \ldots, X_{n}$ where the sum is taken over all terms such that $\Sigma i_{j}=n+1$ with $1 \leqslant i_{j} \leqslant n$.

Proof. Using Theorem 7 in place of Corollary 3 this is the same as the proof of Theorem 4.

In contrast to the commutative case, the above extension is far from unique. Using the following lemma we can give another extension that also can be simply expressed.

Lemma 9 [4, Proposition 1, p. 7]. Let $1, x_{1}, x_{2}, \ldots, x_{n}$ be an SDP in a Hopf algebra $H$. Define $K_{1}, K_{2}, \ldots, K_{n+1}$ inductively by letting $K_{1}=0$ and $K_{i}=\sum_{j=1}^{i-1} x_{i-j}\left(j x_{j}-K_{j}\right)$. Then $(n+1)^{-1} K_{n+1}$ will extend $1, x_{1}, x_{2}, \ldots, x_{n}$.

THEOREM 10. Let $H_{n}$ be the Hopf algebra of Theorem 8. Then

$$
P_{n+1}=\sum(-1)^{m} \frac{i_{m}}{n+1} X_{i_{1}} X_{i_{2}} \cdots X_{i_{m}}
$$


extends $1, X_{1}, X_{2}, \ldots, X_{n}$ where the sum is taken over all terms such that $\sum i_{j}=n+1$ with $1 \leqslant i_{j} \leqslant n$.

Proof. We show by induction that for $n \geqslant 2$ the $K_{n}$ in the lemma is $n P_{n}$ as defined above. First, $K_{2}=X_{1}\left(X_{1}-0\right)=X_{1}^{2}$. As $P_{2}=\frac{1}{2} X_{1}^{2}$, when $n=2$ the theorem is correct.

Assume that the theorem is true for $s \leqslant n$. Then $K_{n+1}=\sum_{j=1}^{n} X_{n+1-j}\left(j X_{j}\right.$ $-j P_{j}$ ). Thus the coefficient of $X_{n+1-j} X_{j}$ is $j$ and by induction (if $m>2$ ) the coefficient of $X_{i_{1}} X_{i_{2}} \cdots X_{i_{m}}$ is $(-1)(-1)^{m-1} i_{m}$.

Of course, by symmetry, there is another extension with coefficients $(-1)^{m} i_{1} /(n+1)$.

Note. Theorem 4 can also be derived from either Proposition 5 or Theorem 10.

\section{BIBLIOGRAPHY}

1. K. Newman, Constructing sequences of divided powers, Proc. Amer. Math. Soc. 31 (1972), 32-38.

2. Sequences of divided powers in irreducible, cocommutative Hopf algebras, Trans. Amer. Math. Soc. 163 (1972), 25-34.

3. K. Newman and M. Sweedler, $A$ realization for the additive Witt group (to appear).

4. B. Shay, Polynomial-dual coalgebra structure in Hopf algebras, Ph.D. Thesis, CUNY, New York, 1972.

12 Stanford Place, Champaign, Illinois 61820 (Current address of A. J. Berkson)

Department of Mathematics, University of Illinois at Chicago Circle, Chicago, ILLINOIS 60680

Current address (Kenneth Newman): Department of Mathematics, University of Massachusetts, Boston, Massachusetts 02125 